\title{
Sprachontologie und Entwicklung natürlicher Sprachen
}

\author{
Linguistic ontology and development of natural languages
}

\author{
Michail L. KOTIN \\ Universität Zielona Góra/ University of Zielona Góra \\ E-mail: michailkotin1@gmail.com
}

\begin{abstract}
The real existence of human languages is, according to Prof. Franciszek Grucza, an anthropocentric phenomenon, i.e., it concerns not only the individual speaker's competence in the Chomskyan model, but also the existence of a language as instrument of mental activity and communication, which is situated, as an idiolect, in the minds of human beings who belong to the given language community. Idiolects are, for their part, phenomena underlying mutual changes caused by the development of the individual awareness in its permanent interaction with the idiolects of other members of the language society. The detection of these changes and their appropriate interpretation allows us to draw conclusions concerning the interaction of ontogenesis and phylogenesis in the creation of human languages. On the background of the anthropocentric linguistics developed by F. and S. Grucza, the problem in question receives a new dimension, namely it is about the dynamic aspect of the interaction of individual and socially-conditioned features as well as language acquisition and so called "systemic" change in the process of language evolution.
\end{abstract}

Keywords: anthropocentric language concept, origin of language, language change, phylogenesis, ontogenesis, language contacts

\section{Einleitung. Ontologie und Genealogie der Sprache im Spiegel der anthropo- zentrischen Linguistik}

Das Wesen der natürlichen Sprachen besteht nach der Kernthese der Warschauer Schule anthropozentrischer Linguistik (vgl. F. Grucza 1997: 11, S. Grucza 2011: 151) darin, dass sich natürliche menschliche Sprachen als Instrumente des Denkens und Kommunizierens in Form von sog. Idiolekten im Bewusstsein des jeweiligen Individuums befinden, welches zu einer bestimmten Sprachgemeinschaft gehört. Die Tradition dieser Sprachbehandlung ist sehr reich und geht u.a. auf J.N. Baudouin de Courtenay (1922: 3) zurück. Laut seiner Konzeption gibt es nämlich so viele konkrete polnische, deutsche etc. Sprachen, wie viele Köpfe von Polen, Deutschen etc. es gibt. Konkrete Individuen, die er als ,sprachliche Menschen” bezeichnet, sind Träger und „Objektivierer” ihres individuellen Sprachdenkens. Sie kommen zur Welt und sterben, und mit ihnen entstehen und verschwinden auch individuelle Sprachen. Dies ist der Grund dafür, dass sich die reale Zusammensetzung einer Sprachgemeinschaft in ständiger Bewegung befindet, sodass Heraklits berühmte Worte $\Pi \dot{v} v \tau \alpha \dot{\rho} \varepsilon \tilde{i}$ (,Alles fließt, alles ist in ständiger Bewegung, alles ist vergänglich“) auch - ja vor allen Din- 
gen - die Sprache(n) betrifft. Die Leitthese der Warschauer Schule anthropozentrischer Linguistik erkennt einem Menschen die Rolle des Trägers mehrerer Sprachen als in seinem Bewusstsein lebenden Idiolekten - zu. Dazu gehört in erster Linie seine Muttersprache, welche aber je nach dem konkreten Bereich ihrer Anwendung in Subidiolekte zerfällt; aber auch sämtliche Fremdsprachen, die ein Mensch beherrscht, machen den unveräußerlichen Bestandteil seiner ,idiolektalen Sprachfähigkeit“ aus. Die sprachliche Ontogenese, d.h. die Aneignung und Entwicklung der individuellen Sprachkompetenz eines Kindes, multipliziert sich bei Tausenden Sprachträgern, die in deren Totalität eine sich ständig entwickelnde und verändernde Sprachgemeinschaft bilden. Der schriftlich übertragene Nachlass einzelner Gemeinschaften als Gesamtheit von individuell produzierten Texten gestatten es einem Philologen bzw. Linguisten, die Mechanismen des Sprachwandels zu ermitteln. Für vorschriftliche Epochen kann dies auf Grund von internen und externen Rekonstruktionen erfolgen. Die Kongenialität von Ontogenese und Phylogenese ist dabei zwar keine mechanistische Wiederholung der Mikroentwicklung bei der Makroevolution, welche die „Muse Klio" aus der Theorie des Sprachwandels definitiv verbannen ließe - wie dies u.a. D.W. Lightfoot (1999) fordert -, aber ihre Missachtung führt zu immensen Verlusten bei den Versuchen, den Sprachwandel adäquat und suffizient zu erklären. Der vorliegende Beitrag setzt sich zum Ziel, die Fragen des Sprachwandels auf dem Hintergrund der theoretischen Prämissen des anthropozentrischen Herangehens an die Sprachevolution zu behandeln. Dabei stehen die Beziehungen zwischen Ontogenese und Phylogenese im Mittelpunkt der Erwägungen.

\section{Das Problem des Sprachursprungs: Ontogenese und Phylogenese}

Heute ist es eher unüblich, den Sprachwandel mit dem Sprachursprung in Verbindung zu bringen. Dafür gibt es einige Gründe, die sich sowohl aus der Geschichte der Sprachwissenschaft ergeben als auch gemeinhin ontologischer Natur sind, d.h. das Wesenhafte an der menschlichen Sprache selbst betreffen.

Im Bulletin de la Societé de Linguistique de Paris für das Jahr 1871 wurde der Beschluss der Pariser Linguistischen Gesellschaft veröffentlicht, keine linguistischen Forschungsprojekte mehr zu fördern, ja gar zu bewerten, welche sich mit Fragen des Ursprungs natürlicher menschlicher Sprachen befassen. Dabei waren gerade diese Probleme ein Jahrhundert davor das Thema lebhaftester Diskussionen. Hier genügt zu erwähnen, dass die Preußische Akademie der Wissenschaften im Jahre 1770 eine Preisfrage zur besten Erklärung des Sprachursprungs ausgeschrieben hat. Im damaligen Streit zwischen J.P. Süßmilch und J.G. Herder hat sich der Letztere mit seiner These vom menschlichen Ursprung der Sprache gegen Süßmilchs These von ihrer göttlichen Herkunft durchsetzen können. Aber nicht nur die Lösung des Problems, sondern sogar seine Stellung war hundert Jahre später schon Geschichte. Die sich immer stärker als positivistisch definierende Wissenschaft (darunter auch Sprachwissenschaft) wollte nämlich nichts mehr mit Gegenständen zu tun haben, die sich nicht mithilfe empirischer Methoden entweder verifizieren oder falsifizieren ließen. Ch.F. Hockett (1960: 5) sieht darin zu recht ein Indiz der Zeit: „This action was a symptom of the times. Speculation about the origin of language had been common 
throughout the 19th century, but had reached no conclusive results". Viel früher hat die Französische Akademie übrigens eine ähnliche Entscheidung getroffen, die viel bekannter ist als der Beschluss der Pariser Linguistischen Gesellschaft. Es handelt sich um den Verzicht der Akademie, neue Projekte eines perpetuum mobile zu bewerten. Obwohl zum Zeitpunkt dieser Entscheidung der Grund für eine prinzipielle Unrealisierbarkeit dieser Idee (also der Energieerhaltungssatz) noch nicht entdeckt wurde, hat man einfach unter dem Druck negativer Resultate darauf verzichtet, sich mit weiteren Vorschlägen eines ,ewigen Triebwerks“ zu befassen. Bezüglich der Versuche, Erscheinungen zu erforschen, welche sich unmittelbarer empirischer Zugriffe entziehen, hat sich die positivistische Wissenschaft generell von der Faustregel leiten lassen, die der englische Philosoph und Präsident der Londoner Philosophischen Gesellschaft A. Ellis im Jahre 1873 formuliert hat: Untersucht werden muss nur das, was tatsächlich existiert (vgl. J.H. Stam 1976, 259).

Dennoch rückte das Problem des Ursprungs natürlicher Sprachen - wenngleich in etwas anderer Form - im 20. Jh. wieder in den Mittelpunkt des Forschungsinteresses. Es wurde nämlich mit Erklärungsansätzen für Sprachwandel in Verbindung gebracht. Die rasche Entwicklung strukturalistischer Methoden der Spracherforschung seit de Saussure, die zunächst zur „synchronen Wende“ führte, hat etwas später auch die diachrone Dimension der Sprache in Mitleidenschaft gezogen. Dabei musste u.a. zur evidenten Kollision zwischen Sprachwandel und Sprachursprung Stellung genommen werden, da es sich in beiden Fällen um dynamische Prozesse in der Sprachevolution handelte, die einer theoretischen Reflexion bedurften. Zunächst wurde diese Kollision auf die denkbar minimalistische Art und Weise aufgelöst. O. Jespersen $(1922,418)$ formuliert nämlich sehr deutlich: , [...] transformation is something we can understand, while a creation out of nothing can never be comprehended by the human understanding." Ein halbes Jahrhundert später wurde diese skeptische Feststellung von einem der führenden Vertreter des Neodarwinismus in der Linguistik R. Lass (1997, 305) wie folgt kommentiert: „I'm not sure that this is true, but whether it is or not there is an interesting theoretical problem". In der Tat ist das Problem des Sprachursprungs gerade aus ontologischer und genealogischer Sicht höchst interessant. Das Paradoxe daran ist dabei nicht nur - und nicht vorrangig - die Tatsache, dass die Herkunft sämtlicher Naturphänomene lediglich eine relative Erklärungsadäquatheit vorsieht. Die anthropozentrische Sprachbehandlung, welche per definitionem die Lokalisierung der Sprache als Sondermodul im menschlichen Bewusstsein, also im menschlichen Gehirn ansetzt, erkennt ihr somit einen ontologischen sowie genealogischen Sonderstatus zu. Dies wurde von M.K. Mamardašvili und A.A. Pjatigorskij (2011: 24-9) sehr präzis auf den Punkt gebracht. Die Erklärung des Sprachursprungs in der Ontogenese und somit auch in der Phylogenese mithilfe einer üblichen Theorie sei nicht möglich, da eine übliche Theorie die Forschungsobjekte erkläre, welche sich außerhalb des sie erforschenden Subjekts befinden. Doch im Fall der - anthropozentrisch definierten (M.K.) - menschlichen Sprache hätten wir, ähnlich wie bei Phänomenen wie unser Bewusstsein oder z.B. unser Tod, mit einem Objekt zu tun, welches unmittelbar zur Sphäre des Bewusstseins gehöre, sodass jede Theorie aus der Sicht des Subjekts notgedrungen tautologisch sei. Daher sei hier nicht eine Theorie, sondern 
vielmehr eine Metatheorie erforderlich. Bezüglich des Sprachursprungs stellen sie in diesem Zusammenhang zu Recht fest, die genetisch primäre Voraussetzung der Sprache sei endgültig verschwunden und kann nicht mehr rekonstruiert werden (vgl. M.K. Mamardašvili/ A.A. Pjatigorskij 2011: 26), da das Subjekt, welches über seine Sprache reflektiert, diese schon besitzt und keinen objektiven Zugang zum postulierten „vorsprachlichen“ Zustand hat (ibid.). Diese Feststellung ist nun unbestritten anthropozentrisch, da es sich hierbei um die Reflexion eines Individuums bezüglich der Entstehung seiner Kompetenz handelt, welche dieses Individuum von dem Zustand unterscheidet, als es noch nicht reflexionsfähig gewesen ist. Man kann über den „ontologischen Status“ dieses Individuums, einschließlich der Frage, ob es im „vorsprachlichen Stadium" seiner Entwicklung überhaupt als Individuum definierbar sei, lediglich spekulieren. Alle Versuche, den Moment der Entstehung der Sprachkompetenz beim Kind (Ontogenese) oder bei der Menschheit (Phylogenese) zu fixieren, schlagen aber gerade wegen der Unmöglichkeit, ,sprachliches Subjekt“" vom ,,sprachlichen Objekt" zu trennen, fehl.

Das Gesagte bedeutet keinesfalls die prinzipielle Abwegigkeit eines jeden Erklärungsansatzes zum Sprachursprung. Löst man sich von der ,individuell reflektierten“ Ontogenese (und somit von der anthropozentrischen Methode) los, kann freilich darüber spekuliert werden, wie die menschlichen Sprachen als ,beobachtbare Zeichensysteme“ hätten entstanden sein können. Allerdings erhält man dabei kein konsistentes Modell der Herkunft natürlicher Sprachen als „,messbare Hirnmodule“ individueller Sprachkompetenzen. Die Kriterien, welche bei derartigen Überlegungen angewandt werden, lassen sich auf zwei wesenhafte Gruppen zurückführen, und zwar (i) ob natürliche Sprachen im Ergebnis einer kontinuierlichen, ununterbrochenen Entwicklung entstehen, und zwar von primitiven Kommunikationssystemen der Lebewesen bis hin zu den Menschensprachen (Kontinuitätshypothese) oder aber ob sie das Ergebnis einer gewissen „Explosion“ in Folge von Mutationen sind, sodass zwischen Tiersprachen und der Sprache der Menschen eine unüberwindbare Kluft liegt (Diskontinuitätshypothese); (ii) ob Sprachkompetenzen angeboren sind oder aber erlernt werden, d.h., ob das Kind bereits mit einem a priori gegebenen Sprachmodul zur Welt kommt (Innate-Hypthese) oder aber ob ihm dieses Modul erst später beigebracht werden muss, damit es sich zumindest eine Sprache aneignen kann (Learned-Hypothese), vgl. die Tabelle unten (laut I. Ulbæk 1998: 31):

\begin{tabular}{|c|c|c|}
\hline $\begin{array}{l}\text { Evolutionary model } \\
\text { Acquisition model }\end{array}$ & Continuity & Discontinuity \\
\hline Innate & $\begin{array}{l}\text { Bickerton, Piker, Ulbanek } \\
\text { and others }\end{array}$ & Chomsky \\
\hline Learned & ,Behaviourism“ & Culturalism \\
\hline
\end{tabular}

Abbildung 1. Learned-Hypothese laut I. Ulbcek (1998: 31).

Für das Chomsky'sche Modell spricht u.a. die Tatsache, dass nach den Ergebnissen der modernen Forschung ein heute geborenes Kind im Prinzip dieselben mentalen 
Eigenschaften besitzt wie ein Neugeborenes am Anfang unserer biologischen Geschichte (vgl. T.V. Černigovskaja 2013: 339). Bezüglich der Sprache hat es zu bedeuten, dass sich das einst im Gehirn entstandene Sprachmodul im Laufe der gesamten Menschheitsgeschichte nicht wesentlich verändert hat, sodass ein vor ca. 50 oder gar 100 tausend Jahren geborenes Kind heute die Fähigkeit besäße, jede moderne Sprache zu erlernen. Die auf Chomsky zurückgehende Konzeption des Sprachursprungs setzt voraus, dass die menschliche Sprache prinzipiell anders beschaffen ist als das, was man normalerweise als „Tiersprachen“ bezeichnet und daher nicht aus den Letzteren im allmählichen Evolutionsprozess entstanden ist. Die natürliche Sprache des Homo sapiens ist somit keine neue, qualitativ höhere und quantitativ umfangreichere Stufe eines kontinuierlich fortschreitenden, seinem Wesen nach einheitlichen und konsistenten Kommunikationscodes von Lebewesen, sondern eine grundsätzlich neue Kompetenz, die darauf beruht, dass die potentielle Nominalphrase mit der potentiellen Verbalphrase als logisches Modul zu einem potentiellen Satz zusammengefügt werden (vgl. N. Chomsky 1957, 1965, 1986). Diese Funktion kann aber keinesfalls mithilfe des Kontinuitätsansatzes erklärt werden und bedarf daher der Annahme eines diskreten „Ur-sprungs“ der Sprachfähigkeit beim Menschen.

Das Gesagte betrifft nun unmittelbar den Zusammenhang zwischen Genealogie der menschlichen Sprache und ihrer Primärfunktion. Postuliert man nämlich, dass die natürliche menschliche Sprache (d.h. eigentlich die Sprachfähigkeit des Homo sapiens) sowohl angeboren ist als auch diskontinuierlich, im Ergebnis einer explosionsartigen Mutation entstanden ist, muss man zugleich zugeben, dass die Sprachfähigkeit nicht nur, ja nicht primär auf das kommunikative Bedürfnis zurückführbar ist, sondern vielmehr Kognition im weiteren Sinn, also einschließlich Orientierung und Reflexion, zum Ausgangspunkt hat. Aus einer anderen Perspektive wird die Sprachontologie äußerst treffend vom berühmten russischen Literaturwissenschaftler und Kulturtheoretiker M. Bachtin (1979: 245) beschrieben. Die Sprache entspringe nach seiner Auffassung vordergründig dem Bedürfnis des Menschen, sich zu äußern, sich zu objektivieren. Sie benötige in ihrem Wesen nur den Sprecher und den Gegenstand seiner Äußerung; wenn die Sprache darüber hinaus als Kommunikationsmittel dienen kann, sei dies lediglich ihre Nebenfunktion, welche ihr Wesen nicht betreffe. Bei der Sprachbehandlung sollten freilich weder die Sprachgemeinschaft noch die Anzahl der Sprechenden geschmälert werden, doch bei der Definition der Natur der Sprache müsse stets beachtet werden, dass dieser Aspekt weder essentiell noch überhaupt unentbehrlich sei. Offenkundig ist diese Sprachauffassung völlig affin sowohl mit den Daten von J. Piaget (1923: 21, 77, 86) hinsichtlich der Egozentrik der sprachlichen Tätigkeit eines Kleinkindes als auch mit Chomskys Verständnis sprachlicher Ontogenese (vgl. N. Chomsky 1996: 30).

Damit müsste man sich bei den Überlegungen zum Ursprung der menschlichen Sprache begnügen, da weitere Spekulationen am Paradoxon von Subjekt und Objekt der Erforschung natürlicher Sprachen scheitern, welches oben bereits dargestellt wurde. Bezüglich des Sprachwandels können allerdings weitere, wiederum primär anthropozentrisch konzipierte essentielle Fragen behandelt werden. 


\section{Das Problem des Sprachwandels: Ontogenese und Phylogenese}

\subsection{Vorbemerkung}

In der Auseinandersetzung mit dem vorwiegend synchron orientierten linguistischen Strukturalismus hat E. Coseriu in seinem 1958 veröffentlichten einschlägigen programmatischen Werk „Synchronie, Diachronie und Geschichte“. Das Problem des Sprachwandels“ (vgl. E. Coseriu 1958/1974) folgende historisch begründete Eigenschaften der Sprache genannt: (i) die Sprache ist ein in Zeichenform im Gehirn der Sprecher lokalisiertes und systematisiertes, sich ständig veränderndes Wissen; (ii) sie ist ein mit der historischen Entwicklung der sie besitzenden und nutzenden Menschengemeinschaft zusammenhängendes historisches Phänomen, dessen Wandel ihr unveräußerliches Merkmal ist und als solches keiner äußeren Ursachen bedarf; (iii) die Sprache verändert sich einfach deshalb, weil sie in der Zeit existiert, genauer, weil sich ihre Träger verändern; (iv) sämtliche Veränderungen sind ihrem Wesen nach das Ergebnis von atomaren, diskreten Akten als Folge der Sprachtätigkeit der Menschen und nicht im Zuge eines allmählichen, kontinuierlichen Wandels; (v) Jeder konkrete Wandel umfasst zwei getrennte Schritte: (a) initiierte Veränderung einer Sprachform bzw. ihrer Bedeutung oder Funktion etc. und (b) Akzeptanz oder Verwerfung des „vorgeschlagenen“ Wandels durch andere Vertreter der jeweiligen Sprachgemeinschaft (im letzteren Fall muss von nicht gelungenem bzw. nicht erfolgtem Sprachwandel gesprochen werden); (vi) die Frage nach universellen Gründen des Sprachwandels ist sinnlos, da sämtliche in der Zeit existierenden Phänomene sich ständig verändern; (vii) durchaus begründet ist dagegen die Frage nach den Ursachen eines konkreten Form- oder Bedeutungswandels in der Sprache (vgl. E. Coseriu 1958/1974: 127ff., 151).

Was verursacht bzw. beschleunigt (oder verlangsamt) nun den konkreten Sprachwandel? Hier wird in der einschlägigen Literatur üblicherweise zwischen „internen“ und ,externen“ "Gründen unterschieden. Zu den ersten gehört sog. ,systembedingter“ Wandel, dessen Ursachen im Sprachsystem selbst bzw. in den Tendenzen seiner Entwicklung liegen; die letzteren sind dagegen außersprachlicher Natur und lassen sich etwas grob und allgemein als sozialhistorische, kulturhistorische und individuelle Faktoren (Einfluss einzelner Personen) einordnen. Die anthropozentrische Sprachbehandlung relativiert allerdings kraft ihres Grundansatzes - absolut zu Recht - diese traditionelle Dichotomie der diachronen Sprachauffassung. Es handelt sich wohl weder um systeminterne (rein linguistische) noch um systemexterne (von außen wirkende) Faktoren, sondern lediglich um unterschiedlich feststellbare Gründe. Ein Teil davon lässt sich nämlich dermaßen genau fixieren, dass die Wirkung einer konkreten Person oder einer sozialen Schicht etc. unmittelbar nachvollziehbar ist. Andere Veränderungen zeigen sich dagegen zunächst als Tendenz, die mehrere Generationen der Sprachträger überdauert und somit den Wandel vom Zustand A zum Zustand B lediglich „evolutionär“ beschreiben lässt, ohne dass individuelle oder soziale Faktoren ermittelt werden können. Dies ist aber letztendlich nur Frage des jeweiligen Erklärungsmodus. Im Weiteren werden aber die jeweiligen Sprachwandelkonzeptionen aus „technischen“ Gründen getrennt behandelt. 


\subsection{Systembedingter interner Wandel}

Wie auch bei der Frage nach dem Sprachursprung, wird die Phylogenese auf dem Hintergrund der Ontogenese betrachtet. D.W. Lightfoot (1999) hat die Ontogenese sogar dermaßen hoch rangiert, dass der Phylogenese überhaupt kein Raum mehr gelassen wurde, und die „Muse Clio" aus der Theorie des Sprachwandels nach seiner Meinung vollkommen aus der Linguistik verbannt werden könnte, was schon oben kurz behandelt wurde. Freilich ist Lightfoots Anspruch zu apodiktisch und in vielem unbegründet, aber die Rolle der Beobachtung des ,individuellen Wandels“ bei der Aneignung der Sprache durch das Kind darf keinesfalls geschmälert werden. D.W. Lightfoot stellt nämlich zu Recht fest, dass die Veränderung in den grundlegenden Algorithmen der Normbehandlung bei sprachlicher Akquisition durch Kinder unumgänglich dazu führen, dass gewisse „Fehler“ als Normverstöße, welche jedoch durchaus systemkonform sind, dazu führen, dass die Fehler von heute zu den Normen von morgen werden. Dieser Erklärungsansatz geht unumstritten mit der anthropozentrischen Maxime einher, dass vermittels der Sprache im Gehirn eines Individuums das Wissen in Zeichenform nicht nur akkumuliert, sondern auch transformiert und ggf. verändert wird. Die Form der Sprachzeichen selbst unterliegt dabei einem Wandel, welcher ein Optimierungsszenario für die Harmonisierung der Form-Funktion-Korrelation umsetzt. Der Sprachwandel ist demnach nichts anderes als eine neue Parametersetzung bei bleibenden Prinzipien der Organisation der Tiefenstruktur potentieller Sätze im Sinne des von N. Chomsky und seiner Schule konzipierten Prinzipien-Parameter-Modells (vgl. N. Chomsky/ H. Lasnik 1993, D.W. Lightfoot 1991). Durch Anhäufung und Polarisierung von Varianten sowie Durchsetzung von denjenigen davon, welche die alten Normen durch Neuordnung bei Parametersetzung gegen neue ersetzen, erfolgt nun mit der Zeit auch eine Neudefinierung von Prinzipien. Im Ergebnis entstehen neue Sprachformen, die ihrerseits eine Basis für weiteren Wandel bilden.

G. Fanselow und S. Felix (1/1987) stellen ein Wandelmodell auf, bei dem das Sprachbewusstsein ständig auf der Suche nach neuen, ökonomischeren Ausdrucksformen ist und somit Optimalitäten erreicht, die ihrerseits durch weitere Ökonomisierung optimiert werden (vgl. auch E. Ronneberger-Sibold 1980). Im Grunde genommen ist ihr Modell durchaus mit dem von D.W. Lightfoot affin, auch wenn ihre Deskriptionen der Sprachentwicklung auf Grund objektiver Parameter erfolgen und daher eher phylogenetisch als ontogenetisch konzipiert sind. Doch das Ökonomieprinzip an sich ist sowohl aus ontogenetischer als auch aus phylogenetischer Sicht mehr als problematisch. Die Sprachaneignung durch das Kind demonstriert ja an ihrer Oberfläche mustergültige Prozesse von Komplikationen und nicht von „Vereinfachungen“ verschiedener Art. Auch bezüglich der historischen Entwicklung natürlicher Sprachen wäre zunächst der naive Gedanke als richtig einzuschätzen, dass sich die Sprache von ersten primitiven Formen hin zur immer größeren Komplexität entwickeln sollte. Auch die Behandlung der Sprache als Tätigkeit im Kommunikationsprozess schließt zumindest eine linear verstandene Ökonomie als Grundprinzip der Kommunikation eher aus: Es geht nämlich wohl kaum um Umsetzung ökonomischer Ausdrucksweisen um jeden Preis, sondern vielmehr um effizientes Kommunizieren, welches sowohl ökonomisch als auch sogar extrem unökonomisch verlaufen kann, je nachdem, welche konkreten 
Kommunikationsziele gesetzt werden bzw. wie sie am besten erreicht werden können (vgl. R. Keller 2003). Bei der Frage nach der Herkunft der Sprache ist das Ökonomieprinzip in gewisser Hinsicht , janusköpfig“. Freilich konnten die natürlichen Sprachen nicht ,von Grund auf“ dermaßen unökonomisch gewesen sein, dass ihre weitere Entwicklung einzig und allein dem Ökonomieprinzip folgte. Da nun aber diese Entwicklungsstufe der Sprachen nicht mehr rekonstruiert werden kann, können nur schon vorhandene, d.h. schriftlich bezeugte, Sprachstufen zum Vergleich gezogen werden. Diese weisen meist recht komplexe Deklinations- und Konjugationsparadigmen auf, welche sich bei der weiteren Entwicklung der älteren Sprachen auf den ersten Blick tatsächlich vereinfacht haben, auch wenn hier „Vereinfachung“ lediglich nur sehr allgemein zu verstehen ist. Nichtsdestoweniger könnte man z.B. die Ausbildung analytischer Sprachformen im Vergleich zu synthetischen als Ausdruck der Sprachökonomie interpretieren, falls man tiefere Relationen zwischen der Komplexität verschiedener Sprachebenen schmälert.

Die Theorie der ,linguistischen Natürlichkeit“ (vgl. W.U. Wurzel 1984, W. Mayerthaler 1981, W.U. Dressler et al. 1987) versucht nun eine Brücke zwischen Sprachökonomie, Komplexität und Optimalität zu schlagen. Zunächst führt sie hierzu den Begriff der Markiertheit ein, welcher sich jedoch von der gängigen, von dem Prager Zirkel geprägten Markiertheit bzw. Merkmalhaftigkeit unterscheidet. Als markiert werden nämlich morphologische Formen verstanden, die die jeweiligen grammatischen Kategorialfunktionen mehr als minimal kodieren und somit nicht natürlich seien, da die morphologische Natürlichkeit nach der Faustregel dieser Theorie eine Eins-zu-Eins-Entsprechung (Ikonizität) von Form und Funktion voraussetzt. So seien die Formenpaare Tisch : Tisch-e, Kind : Kind-er, Frau : Frau-en maximal natürlich, da hier die Mehrzahl jeweils ikonisch durch ein zusätzliches morphologisches Element, das Pluralsuffix, gekennzeichnet ist. Die Pluralformen des Typs Buch : Bücher sind nicht mehr so ikonisch und somit weniger natürlich, da sie sozusagen formal „doppelt belegt" sind, und zwar neben dem Pluralsuffix die Umlaut-Alternation des Stammvokals aufweisen. Dagegen wäre z.B. das Paar Vater : Väter zwar nicht so natürlich wie Tisch : Tisch-e etc., aber natürlicher als Buch : Büch-er, ist ja der Plural, wenngleich wurzelintern und nicht wurzelextern (wie im klassischen Fall der Ikonizität) indiziert, so doch nur einmal belegt. Nicht ikonisch sind dagegen Paare wie u.a. Koffer : Koffer, wo der Plural lediglich aus dem Kontext ermittelt werden kann, und Formenpaare des Typs Eltern-teil : Eltern werden als kontraikonisch bezeichnet, da hierbei die Singularform und nicht die Pluralform suffixal indiziert ist. Die Grundidee der Theorie der linguistischen Natürlichkeit in deren diachronischem Ausmaß besteht nun darin, dass sich die Sprachen in Richtung auf stärkere Ikonizität entwickeln und un- bzw. kontraikonische Markierungen zunehmend zugunsten ikonisch-natürlicher Formenkodierung abbauen.

Wie lassen sich nun Minimalitäts-, Optimalitäts- bzw. Natürlichkeitskonzepte aus ontogenetischer Sicht bewerten? Es kann hier kein eindeutiges Urteil gefällt werden, zumal die experimentell feststellbaren Daten keine Kongenialität von Ontogenese und durch diese Theorien postulierte Phylogenese aufweisen. Hierzu nur ein - dabei aber sehr aussagekräftiges - Beispiel. In der jüngeren Forschung der Sprachaneignung 
durch Kinder (untersucht wurden Kinder mit Englisch als Erstsprache) ist - gegen Erwartungen - nachgewiesen worden, dass das Erlernen von irregulären Konjugationsformen des Typs come : came früher erfolgt als die Aneignung der Fähigkeit, reguläre Formen richtig zu bilden, wobei es aber erst später zur Übergeneralisierung des regulären Musters vom Typ spell : spell-ed kommt, sodass die Formbildung des Typs *com-ed oder *cam-ed entsteht (vgl. L. Bornkessel-Schlesewsky/ M. Schlesewsky 2009: 47-48). Dagegen werden irreguläre Formen nur selten übergeneralisiert (ibid.: 48). Ohne hier auf neuroanatomische Erklärungen dieser Phänomene einzugehen, welche eine Arbeitsteilung verschiedener Hirnareale bei der Bildung regelgesteuerter und gedächtnisbasierter Flexionsformen postuliert (ibid.: 49-57), kann festgehalten werden, dass gerade irreguläre Formen sozusagen „ontogenetisch primär“ sein können. Dies geht völlig einher mit der phylogenetischen Evidenz, dass z.B. die germanischen starken Verben primäre Verbalstämme mit indogermanischem Ablaut darstellen, wohingegen die von diesen Verbalstämmen oder aber von Substantiv-, Adjektiv- Adverbialstämmen etc. abgeleiteten regulären (,schwachen“) Verben erst später gebildet wurden. Freilich werden dabei viel kompliziertere Mechanismen bemüht als dies eine einfache unidirektionale Entwicklung zu Regularität und schwächerer Markiertheit im Rahmen diverser Optimalitätsszenarien vorsieht.

Die neudarwinistischen Zugriffe auf Sprachwandel (vgl. u.a. R. Lass 1997) weisen dem Sprachsystem, ähnlich zu lebendigen Organismen, immanente Eigenschaften zu, die einer evolutionär angelegten Analyse zugänglich sind, welche weitgehend unabhängig von der Rolle der Sprachträger erfolgen kann. Die Sprachentwicklung wird dabei als Anpassung an die sprachliche „Umwelt" bzw. an die darin vorhandenen „epigenetischen Landschaften“ (Adaptation) behandelt, während die Form-FunktionBeziehung in ihrer dynamischen Dimension als Anpassung der Sprachform an neue Umweltbedingungen definiert wird. Dieser Funktionswandel wird der Anschaulichkeit halber mit der Adaptation lebender Organismen an die sich verändernden Bedingungen ihrer Umwelt verglichen. So wie die Vogelfedern ihre Funktion von Wärmeerhaltung auf Fluginstrumente ändern, ändern sich laut dieser Theorie die Funktionen des Lautwandels von rein phonetischer Assimilation zur grammatischen Kodierung (vgl. Ablaut und Umlaut in den germanischen Sprachen) etc. Daher sind laut R. Lass (1997: 337) für einen Sprachhistoriker gerade diejenigen Wandelphänomene am interessantesten, die langwierigen, mehrere Generationen der Sprachträger umfassenden Wandel demonstrieren, da sie die unmittelbaren, persönlich initiierten Einflüsse auf die jeweiligen Veränderungen ausschließen. Neben Adaptation werden dabei Erscheinungen untersucht, die - ähnlich zur Biologie - als Exaptation (vgl. St.J. Gould/ E.S. Vrba 1982: 4-15, L. de Cuypere 2005: 13-26, R. Lass 1997: 316-324) bezeichnet werden, d.h. nicht Funktionswandel, sondern Funktionsverlust von Sprachformen, wobei die jeweilige Form auch nach Funktionsverlust weiter bestehen bleibt, wie z.B. Brustwarzen bei Männern, Steißbein, Haare auf der menschlichen Haut, Blinddarmfortsatz etc. In der Sprache werden hier u.a. Folgen von Verners Gesetz (grammatischem Wechsel) bei starken Verben (wie in dt. leiden : litten, ziehen : gezogen etc.) als Beispiele für Exaptation angeführt, in deren Folge laut Lass junks ,(sprachlicher) Müll, Abfall“" (vgl. R. Lass 1997: 316-318) entstehen, die den kognitiven Aufwand 
beim Erlernen der Sprache erhöhen, ohne dass dabei neue Funktionen markiert werden.

Die neudarwinistischen Erklärungen für Sprachwandelmechanismen sind leider generell so konzipiert, dass kein Platz für ontogenetische Parallelen bleibt. Laut Lass sind für den Sprachevolutionsforscher gerade Wandelphänomene von Interesse, die kurzfristigen Wandel ausschließen. Diese These schließt aber nicht nur phylogenetischen kurzfristigen Wandel aus, sondern weitgehend auch den ontogenetischen Wandel (Spracherwerb), da dieser immer kurzfristig, dabei aber ausgerechnet aus evolutionsbiologischer Sicht von essentieller Bedeutung ist.

Gehen wir nun zum mittel- bzw. kurzfristigen Wandel über, der vorwiegend nicht „intern-systematisch“, sondern vielmehr „extern-soziokulturell“" erklärt wird und keine direkten Parallelen in der Ontogenese hat.

\subsection{Sozialhistorisch bedingter externer Wandel}

Diese Wandelart schließt die Untersuchung der Wechselwirkung von Ontogenese und Phylogenese weitgehend aus. Das Einzige, was hier in Frage käme, wären Überlegungen zur eventuellen Kongenialität der Reihenfolge in der Aneignung des neuen Wortguts durch das Kind und der Wortschatzerweiterung einer gegebenen Sprache. Doch auch dieser Aspekt der Sprachdynamik lässt sich wohl kaum befriedigend beschreiben, gibt es ja in der Sozialentwicklung der Gesellschaft zu viele externe Inputphänomene, als dass auch nur sehr allgemein postulierte Affinitäten zwischen individueller Akquisition und Gesamtentwicklung denkbar wären. Daher werden unten lediglich allgemeine, grundsätzlich nur phylogenetisch zu verstehende Gründe für extern bedingten Sprachwandel aufgeführt, die von verschiedenen Forschern genannt werden: (i) Veränderungen in den Ritualen einer gegebenen Sprachgemeinschaft (vgl. W. Labov 1972); (ii) Veränderungen der wichtigsten Lebensformen der Sprachbenutzer, z.B. Wechsel des nomadischen zur sesshaften Lebensweise, Völkerwanderungen etc. (vgl. W.P. Lehmann 1978); (iii) kulturelle und zivilisatorische Entwicklungen: Wissenschaft und Technik, Religion, Recht, Mediensphäre sowie Kriege, Revolutionen etc. (vgl. E. Coseriu 1958/1974); (iv) Einfluss von einzelnen Personen auf die Sprachentwicklung: Theologen, Dichter, Kanzleischreiber, Philologen, Politiker u.a. (vgl. A. Bach 1939); (v) Einfluss von Kulturträgerschichten als führenden Kräften einer Sprachgemeinschaft auf einer bestimmten Etappe ihrer Entwicklung: Mönche, Ritter, Kaufleute, Bürgertum etc. (vgl. H. Eggers 1965-1973).

Auch hier kann die anthropozentrisch ausgerichtete Sprachforschung einen erheblichen Beitrag leisten, falls sie in Kooperation mit der Textlinguistik bzw. den philologischen Methoden der Textforschung den Beitrag einzelner Individuen zur Ausbildung neuer Sprachformen bzw. Umdeutung ihrer genuinen Semantik und Funktion feststellen hilft.

Zuletzt sei hier das Problem des Einflusses der Sprachkontakte auf den Sprachwandel angesprochen. Bekanntlich werden Sprachkontakte als einer der stärksten Faktoren des Sprachwandels betrachtet. Die grundsätzliche Frage ist dabei aber, ob sie ihrem Wesen nach zu externen Wandelfaktoren oder aber zu den Beschleunigern interner Wandeltendenzen gezählt werden müssen. Laut der so genannten ,anything- 
goes"-Hypothese (vgl. Y. Matras 1998, S.G. Thomason/ T. Kaufman 1988, S.G. Thomason 2001) ist im Prinzip jeder Wandel in der ,,aufnehmenden“ Sprache unter dem Einfluss der Kontaktsprache möglich. Mehrere Fallstudien beweisen dagegen, dass zumindest beim systematischen, regelgesteuerten Sprachwandel lediglich Entwicklungstendenzen beschleunigt werden, welche das jeweilige ,aufnehmende“ System deshalb zulässt, weil es selbst vergleichbare Tendenzen, wenngleich in einer nicht dermaßen offenen Form wie die Gebersprache, aufweist (vgl. M.L. Kotin 2012: 319, E. Bidese/ A. Padovan/ A. Tomaselli 2013, 2014, W. Abraham 2014: 444). „Interne“ und ,externe" Wandelfaktoren lassen sich nun auch hier aus anthropozentrischer Perspektive wohl kaum voneinander scharf trennen.

\section{Fazit}

Das anthropozentrische Herangehen an natürliche Sprachen sollte nicht nur aus synchroner, sondern auch aus diachroner Sicht eine stärkere Anwendung finden. Insbesondere ist es gerade bei Fragestellungen produktiv, die Sprachontologie und Sprachgenesis betreffen, und zwar vor allen Dingen in dem für die historische Sprachwissenschaft essentiellen Bereich der Affinitäten von Ontogenese und Phylogenese in der Sprachentwicklung liegen. Dieses Herangehen erlaubt es einerseits bei der Behandlung von Problemen des Sprachursprungs die Grenzen der Erkenntnis auf dem Gebiet der menschlichen Sprachen angemessen festzulegen. Andererseits ist es bei der Erklärung der Wechselwirkung interner und externer Faktoren des Sprachwandels äuBerst hilfreich.

\section{Bibliographie}

Abraham, W. (2014), Schriften zur Synchronie und Diachronie des Deutschen. In: A. Kątny/ M.L. Kotin/ E. Leis et al. (Hrsg.), Danziger Beiträge zur Germanistik. Frankfurt a. M. etc.

Bach, A. (1939): Geschichte der deutschen Sprache. Heidelberg.

Bachtin, M.M. (1979), Estetika slovesnogo tvorčestva. Moskva.

Baudouin de Courtenay, J.N.. (1922), Zarys historii języka polskiego. Warszawa.

Bidese, E./ A. Padovan / A. Tomaselli (2013), Bilingual competence, complementizer selection, and mood in Cimbrian. In: W. Abraham/ E. Leiss (Hrsg.), Dialektologie in neuem Gewand: Zu Mikro-/Varietätenlinguistik, Sprachenvergleich und Universalgrammatik. Hamburg, 47-58.

Bidese, E./ A. Padovan/ A. Tomaselli (2014), The syntax of subordination in Cimbrian and the rationale behind language contact. In: "Language Typology and Universals" 67/4, 489-510.

Bornkessel-Schlesewsky, I./ M. Schlesewsky (2009), Processing Syntax and Morphology: A Neurocognitive Perspective. Oxford.

Bulletin de la Societé de Linguistique de Paris (1871), volume premier. Paris.

Chomsky, N. (1957), Syntactic Structures. The Hague-Paris.

Chomsky, N. (1965), Aspects of the Theory of Syntax. Cambridge (Mass.). 
Chomsky, N. (1986), Knowledge of Language. New York.

Chomsky, N. (1996): Powers and Prospects. Reflections on human nature and the social order. London.

Chomsky, N./ H. Lasnik (1993), Principles and parameters theory. In: J. Jacobs/ A. von Stechow/ W. Sternefeld et al. (Hrsg.), „Syntax. Ein internationales Handbuch zeitgenössischer Forschung“" Halbbd. 1. Berlin/ New York, 506-569.

Coseriu, E. (1958), Sincronia, diacronia e historia. El problema del cambio linguístico. Montevideo. Deutsche Ausgabe: Synchronie, Diachronie und Geschichte. Das Problem des Sprachwandels (1974). München.

Cuypere, L. de (2005), Exploring exaptation in language change. In: „Folia Linguistica Historica. Acta Societatis Linguisticae Europeae” XXVI/ 1-2, 13-26.

Černigovskaja, T.V. (2013), Češirskaja ulybka kota Šrëdingera. Jazyk i soznanije. Moskva.

Dressler, W.U./ W. Mayerthaler/ O. Panagl et al. (1987), Leitmotifs in Natural Morphology. Amsterdam/ Philadelphia.

Eggers, H. (1965-1973): Deutsche Sprachgeschichte in 4 Bd. Reinbeck bei Hamburg.

Fanselow, G./ S. Felix (1987), Sprachtheorie. Eine Einführung in die generative Grammatik, Bd. 1: Grundlagen und Zielsetzungen, Bd. 2: Die Rektions- und Bindungstheorie. Tübingen.

Gould, St.J./ E.S. Vrba (1982), Exaptation - a missing term in the science of form. In: „Paleobiology” 8 (1), 4-15.

Grucza, F. (1997), Języki ludzkie a wyrażenia językowe, wiedza a informacja, mózg a umyst ludzki, In: F. Grucza/ M. Dakowska (Hrsg.), Podejścia kognitywne w lingwistyce, translatoryce i glottodydaktyce. Warszawa, 7-21.

Grucza, S. (2011), Lingwistyka antropocentryczna a badania okulograficzne, In: „Lingwistyka stosowana“ 4, 149-162.

Keller, R. (2003), Sprachwandel - Von der unsichtbaren Hand in der Sprache. 3., durchges. Aufl. Tübingen und Basel.

Kotin, M.L. (2012), Gotisch. Im (diachronischen und typologischen) Vergleich Heidelberg.

Labov, W. (1972), Rules for Ritual Insults. In: W. Labov (Hrsg.), Language in the Inner City, Philadelphia, 297- 353.

Lass, R. (1997), Historical Linguistics and Language Change, Cambridge.

Lehmann, W.P. (Hrsg.) (1978), Syntactic Typology. Studies in the Phenomenology of Language. Sussex.

Lightfoot, D.W. (1991), How to Set Parameters - Arguments from Language Change, Cambridge.

Lightfoot, D.W. (1999), The Development of Language - Acqusition, Change and Evolution. Oxford.

Hockett, Ch.F. (1960), The Origin of Speech. In: "Sceintific American" 203, 88-96.

Mamardašvili, M.K./ A.M. Pjatigorskij (2011), Simvol i soznanije. St. Petersburg.

Matras, Y. (1998), Utterance modifiers and universals of grammatical borrowing. In: "Linguistics" 36/1, 281-331.

Mayerthaler, W. (1981), Morphologische Natürlichkeit. Wiesbaden. 
Piaget, J. (1923), Le language et la pensée chez l'entfant, deutsch von N. Stöber (1983): Sprechen und Denken des Kindes. Frankfurt a.M.

Ronneberger-Sibold, E. (1980), Sprachverwendung - Sprachsystem, Ökonomie und Wandel. Tübingen.

Stam, J.H. (1976), Inquiries into the Origin of Language. The Fate of a Question. New York/ Hagerstow/ San Francisco.

Thomason, S.G. (2001): Language Contact. An Introduction. Edinburgh.

Thomason, S.G./ T. Kaufman (1988), Language Contact, Creolization and Genetic Linguistics. Berkeley/ Los Angeles.

Ulbæk, I. (1998), The origin of language and cognition. In: J.R. Hurford/ M. StuddertKennedy/ Chr. Knight (Hrsg.), Approaches to the Evolution of Language: Social and Cognitive Base. Cambridge/ New York, 30-43.

Wurzel, W.U. (1984), Flexionsmorphologie und Natürlichkeit - Ein Beitrag zur morphologischen Theoriebildung, Berlin. 